DOI: $10.1002 /$ anie. 200600356

\title{
Bioconjugated Ag Nanoparticles and CdTe Nanowires: Metamaterials with Field-Enhanced Light Absorption**
}

\author{
Jaebeom Lee, Tanveer Javed, Timur Skeini, \\ Alexander O. Govorov, Garnett W. Bryant, and \\ Nicholas A. Kotov*
}

The hybrid assembly of inorganic and metallic nanomaterials by means of chemical and biological bonding to yield manifold optical and electromagnetic properties has received widespread attention. ${ }^{[2-11]}$ Currently available highly monodispersed nanomaterials such as semiconductors and noble metals that can be conjugated by ligand-receptor, antigenantibody reactions, polymer tethering, and DNA hybridization are used as building blocks for 2D or 3D superstructures in which new collective properties of these artificial assemblies have been obtained. ${ }^{[12-15]}$ They represent a large class of new materials (i.e., metamaterials) in which the properties are determined not only by classical atomic composition, but also by nanoscale organization of structural components. Recently, metamaterials based on metallic composites have received special attention as these are expected to display negative values for permittivity and refractive index. This property should lead to a multitude of unique optical effects. ${ }^{[11,16,17]}$ Many of these effects are related to surface plasmons on $\mathrm{Au}$ or Ag nanoparticles (NPs), which generate exceptionally high localized electromagnetic fields, and have been exploited in surface-enhanced Raman spectroscopy ${ }^{[18-21]}$ and some optoelectronic devices. ${ }^{[12,22]}$ Metamaterials from semiconductors and their combinations with metals can also produce optical effects that are useful for development of advanced sensing and imaging technologies. ${ }^{[3,24]}$ Recently, we

[*] Dr. J. Lee, Prof. Dr. N. A. Kotov Department of Chemical Engineering

Department of Materials Science and Engineering and

Department of Biomedical Engineering

University of Michigan

Ann Arbor, MI 48109 (USA)

Fax: (+1) 734-764-7454

E-mail: kotov@umich.edu

T. Javed, T. Skeini, Prof. Dr. A. O. Govorov

Department of Physics and Astronomy

Ohio University

Athens, OH 45701 (USA)

Dr. G. W. Bryant

Quantum Processes and Metrology Group

National Institute of Standards and Technology

Atomic Physics Division

100 Bureau Drive, Stop 8423

Gaithersburg, MD 20899-8423 (USA)

[**'] This work was supported in part by NSF Biophotonics and NSF CAREER at UM, and NIST and BNNT initiative at OU.

$\square$ Supporting information for this article is available on the WWW under http://www.angewandte.org or from the author. 
observed that the collective plasmon resonance in a specially designed assembly of Au NPs can stimulate radiative recombination of excitons in CdTe NPs and nanowires (NWs). For example, the bioconjugates of $\mathrm{Au}$ NPs and CdTe NWs assembled with streptavidin (SA) and D-biotin (B) exhibited fivefold enhancement of luminescence and blue shifts of emission bands. Modeling studies showed that the luminescence enhancement originates from amplification of electromagnetic fields induced by $\mathrm{Au}$ NPs in the vicinity of CdTe NWs. ${ }^{[1,25]}$ Other experiments with Au and CdTe NPs in which polymer linkers were used to produce a dynamically conjugated system proved that the interaction between surface plasmons and excitons is a substantial factor in the different ratios of luminescence enhancements obtained. This interaction was dynamically modified by the reversible swelling and deswelling of the polymeric spacers inside the assembled nanoscale superstructures. ${ }^{[26]}$

Herein, we report new superstructures based on $\mathrm{Ag}$ NPs. Although collective interactions between NPs and NWs in the superstructures are also important for $\mathrm{Au}$ NPs, the mechanism for the enhancement of the emission of the Ag-NP conjugates is qualitatively different from that of Au-based NW-NP metamaterials. ${ }^{[1,25,26]}$ In the latter, the emission enhancement comes mostly from the increase of the photon emission that is stimulated by resonance with plasmon oscillations in the NP. However, when $\mathrm{Au}$ is replaced with $\mathrm{Ag}$, the emission enhancement comes from the increase in absorption. A theoretical model for the experiments describes collective plasmon excitations in the Ag-NP shell and provides an accurate explanation of the optical properties of Ag-based NP-NW superstructures. This paper investigates the mechanisms of interactions between NPs and develops the understanding of such hybrid materials, which could be used for a novel class of sensors and actuators with enhanced optical and thermal properties. ${ }^{[1,25]}$

Morphological characterization of each component of the superstructure was carried out by high-resolution transmission electron microscopy (HRTEM) and atomic force microscopy (AFM). From AFM images, the average diameters of $\mathrm{Ag}$ NPs and CdTe NWs were measured to be $3.11 \pm 1.2 \mathrm{~nm}$ and $8.09 \pm 2.3 \mathrm{~nm}$, respectively (see the Supporting Informa- tion). The NWs were assembled from 3.7-nm CdTe NPs according to a procedure described elsewhere (>98\% yield). ${ }^{[27]}$ The NWs had an average length of $1400 \pm 128 \mathrm{~nm}$ (with an aspect ratio of 173) and luminesced at $658 \pm 2 \mathrm{~nm}$. It is known that bulk Ag film has a (111) lattice spacing of $0.2359 \mathrm{~nm}$. The Ag NPs have a lattice spacing of $0.235 \pm$ $0.003 \mathrm{~nm}$, which corresponds to the spacing between (111) planes of Ag crystals. ${ }^{[28]}$ The TEM image showed the Ag NPs attached to the surface or in the vicinity of a CdTe NW to form a fuzzy shell (Figure 1a). There was a noticeable red shift in UV/Vis absorption peak after attachment of proteins to the Ag NPs (Figure $1 \mathrm{~b}, \mathrm{i} \rightarrow \mathrm{ii}$ ); this shift correlates very well with the change of dielectric constant. ${ }^{[29,30]}$
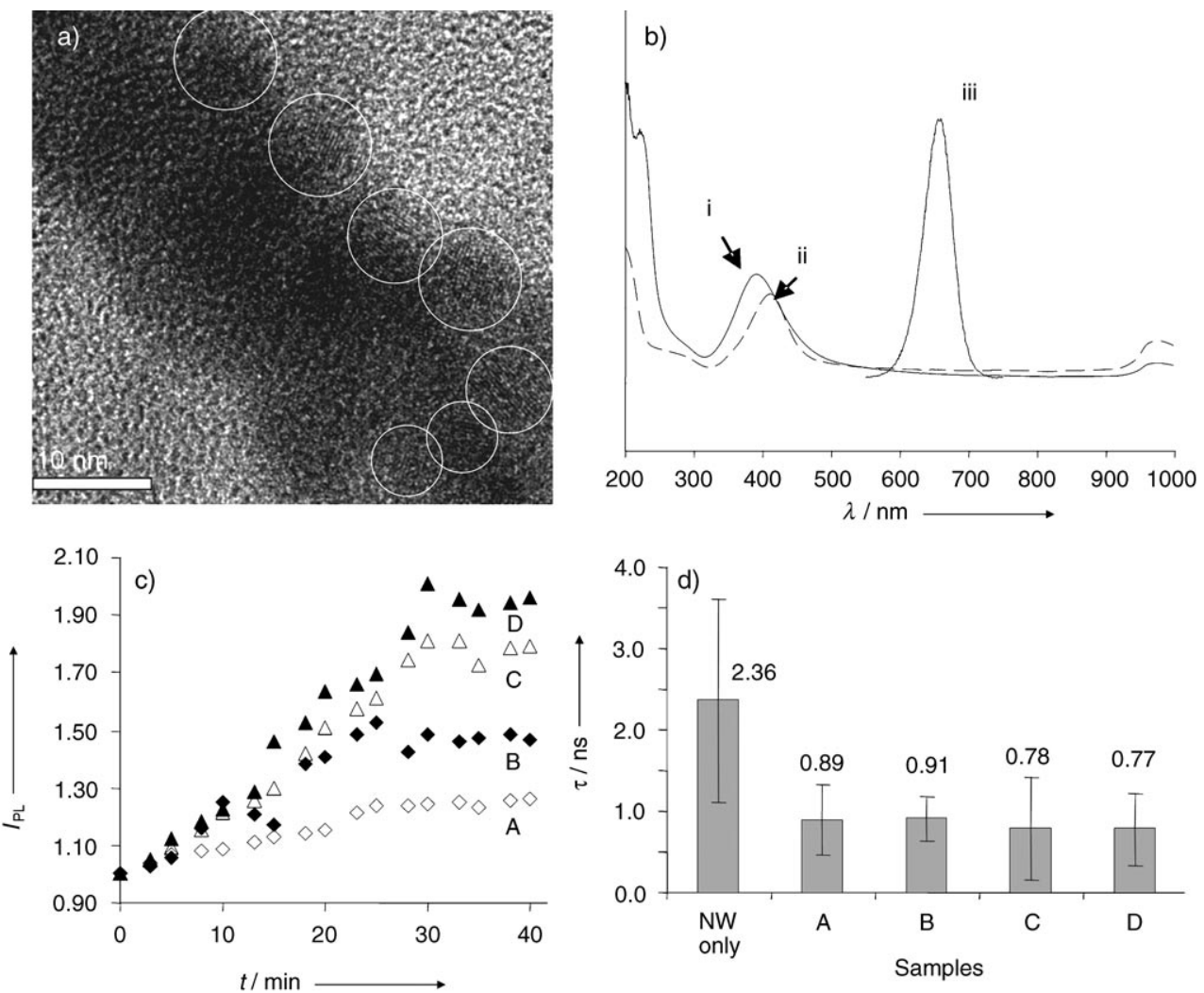

Figure 1. a) TEM image of bioconjugates of NPs and NWs, $300000 \times$, b) UV/Vis and luminescence spectra of Ag NPs and CdTe NWs; i: Ag NPs, ii: Ag NPs with SA, iii: PL of NWs; c) Time courses of the luminescence peak intensities for solutions $A$ to $D$. d) PL lifetimes of the respective samples. 
Table 1: Composition of different NW-NP assemblies prepared from different volume ratios of the stock NW-B and NP-SA dispersions. An averaged NW length of $1400 \mathrm{~nm}$ was used for calculation.

\begin{tabular}{lllll}
\hline & Solution A & Solution B & Solution C & Solution D \\
\hline $\begin{array}{l}\text { volume ratio } \\
\text { (CdTe NW/Au NP) }\end{array}$ & $20: 20$ & $20: 40$ & $20: 80$ & $20: 100$ \\
NP/NW ratio & 4468 & 8936 & 17872 & 22340 \\
\hline
\end{tabular}

$10 \mathrm{~nm}$ during the bioconjugation process; these changes are smaller than those for the analogous reaction with Au NPs (see the Supporting Information). ${ }^{[1]}$ The bioconjugation of ligand receptors with attached nanocolloids was completed in approximately 30 minutes for different molar ratios of CdTe NWs and Ag NPs (Figure 1c). The photoluminescence (PL) lifetime was about $2.36 \mathrm{~ns}$ for the NWs alone, but decreased to around $0.84 \mathrm{~ns}$ for the Ag-conjugated NWs (Figure $1 \mathrm{~d}$ ). The PL intensity after that period was considered the ultimate fluorescence intensity and the assembly process was considered to reach saturation. The kinetics of the luminescence intensity is well-correlated with the conjugation of SA and B involving nanocolloids. ${ }^{[1,31,32]}$ Higher PL intensities at saturation were observed for higher NP/NW ratios (Figure 1c). The spectra of the Ag NP-NW superstructures appear similar to those of $\mathrm{Au} \mathrm{NP}-\mathrm{NW}$ superstructures, and this was initially explained on the basis of exciton-plasmon interactions. ${ }^{[1]}$ However, the underlying mechanism is essentially different. A strong indication of this is that the plasmon-resonance peak of the Ag particles does not overlap in any way with the emission peak of the NWs (Figure $1 \mathrm{~b}$ ), which is required for resonance.

The answer to this apparent discrepancy between the expected mechanism and the experimental results was found in the excitation spectra (Figure 2). As the superstructure

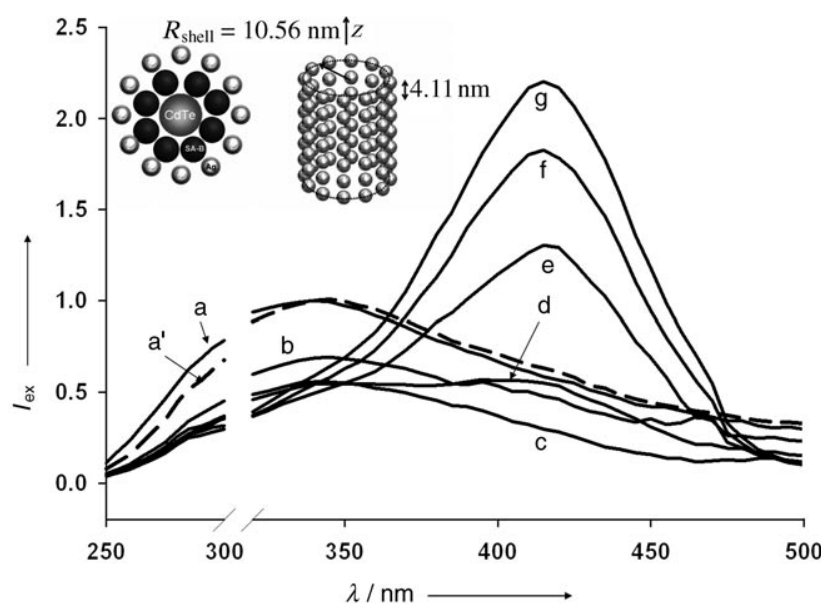

Figure 2. Photoluminescence excitation spectra of conjugated superstructures: spectra of Ag-conjugated NWs recorded every 10 minutes $(\mathrm{a} \rightarrow \mathrm{g})$ for an emission wavelength of $660 \mathrm{~nm}$ (solution D); Auconjugated NWs after 60 minutes ( $a^{\prime}$, dashed line). The gap at $300 \mathrm{~nm}$ corresponds to the strong $\lambda / 2$ peak of the excitation light, removed from the spectra for clarity. Inset: Cross section of the superstructure of Ag NPs, SA-B linkers, and an NW (left). Theoretical model (right) of an Ag shell with periodicity along the cylinder axis. The electromagnetic field is calculated at the center of the Ag-NP shell. forms over a period of 20-30 min, a new feature develops in the vicinity of $420 \mathrm{~nm}$. Importantly, no change is observed at this same wavelength over a period of $60 \mathrm{~min}$ in an experiment with the Au-conjugated NW superstructures under the same conditions (spectrum a'). Firstly, this correlates very well with the plasmon-exciton resonance mechanism suggested previously for $\mathrm{Au} \mathrm{NP}-\mathrm{NW}$ superstructures. ${ }^{[32]}$ Secondly, the excitation peak that develops at $420 \mathrm{~nm}$ for $\mathrm{Ag} \mathrm{NP}-\mathrm{NW}$ superstructures indicates that absorption increases drastically at this wavelength, with the energy eventually channeled into the emission of the NW at $600 \mathrm{~nm}$. Thus, the reason for increase in luminescence of the Ag NP-NW superstructures is stimulated light absorption rather than light emission. The absorption of the CdTe NWs is enhanced at the exciton wavelength as a result of the proximity of the Ag NPs, which have a plasmon band that can oscillate in resonance with the exciton in the semiconductor.

To confirm this hypothesis, we calculated the electric fields inside a superstructure. The theoretical model incorporates an NW, an Ag-NP shell, and SA-B biolinkers (Figure 2, left inset). The radii of the components are taken as follows: $R_{\mathrm{NW}}=4 \mathrm{~nm}, R_{\mathrm{SA}-\mathrm{B}}=2.5 \mathrm{~nm}$, and $R_{\mathrm{AgNP}}=1.56 \mathrm{~nm}$. The corresponding radius of the Ag-NP shell $R_{\text {Shell }}$ is $10.56 \mathrm{~nm}$. The emission intensity of the superstructure is proportional to the factor $P_{\text {emiss }}\left(\lambda_{\text {excitation }}, \lambda_{\text {emiss }}\right)$ [Eq. (1)], whereby $\lambda_{\text {excitation }}$ and $\lambda_{\text {emiss }}$ are the excitation and exciton peak wavelengths and $P_{\text {field }}(\lambda)$ is the electric-field enhancement factor at a particular wavelength [Eq. (2)]. In Equation (2), $E_{0}$ is the amplitude of the external electric field, and $E_{\text {tot }}$ is the resultant field in the center of the Ag-NP shell. The squared resultant electric field is averaged over all solid angles. Although the position of NPs with respect to the NW are assumed to be constant and unaffected by tumbling in solution, this assumption is necessary to account for the fact that the NWs in a solution may have variable orientation with respect to the electric field of incident light.

$P_{\text {emiss }}\left(\lambda_{\text {excitation }}, \lambda_{\text {emiss }}\right)=P_{\text {field }}\left(\lambda_{\text {excitation }}\right) P_{\text {field }}\left(\lambda_{\text {emiss }}\right)$

$P_{\text {field }}(\lambda)=\frac{\left\langle E_{\text {tot }}^{2}\right\rangle_{\Omega}}{E_{0}^{2}}$

Figure 3 shows numerical simulations of the factors $P_{\text {emiss }}\left(\lambda_{\text {excitation, }}, \lambda\right)$ and $P_{\text {field }}(\lambda)$ at the center of the Ag-NP superstructure (Figure 2, right inset) with Maxwell equations (see the Supporting Information). For the curves in Figure $3 \mathrm{~b}$, we fixed the excitation wavelength $\left(\lambda_{\text {excitation }}=420 \mathrm{~nm}\right)$ and varied the emission wavelength $(\lambda)$. The data were obtained for three superstructures with total numbers of NPs $N_{\text {tot }}=56$, 84, and 112. Each superstructure has seven rings, and the inter-ring spacing was taken as $4.11 \mathrm{~nm}$. The corresponding numbers of NPs per ring $\left(N_{\text {ring }}\right)$ were 8,12 , and 16 . Note that the above numbers are less than the maximum possible number of Ag NPs per ring (i.e., 21). The corresponding linear densities of NPs were then calculated as $N_{\text {ring }} / 4.11 \mathrm{~nm}$ $\approx 1.95,2.92$, and $3.89 \mathrm{~nm}^{-1}$. If we now assume that total length of the NW is $1400 \mathrm{~nm}$ and calculate the total numbers of attached NPs using the above linear densities, we obtain 3610 , 
a)

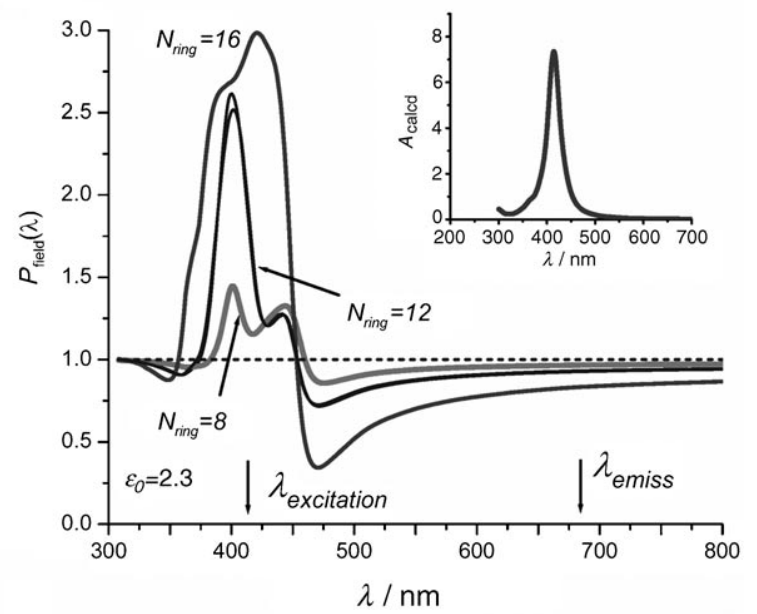

b)

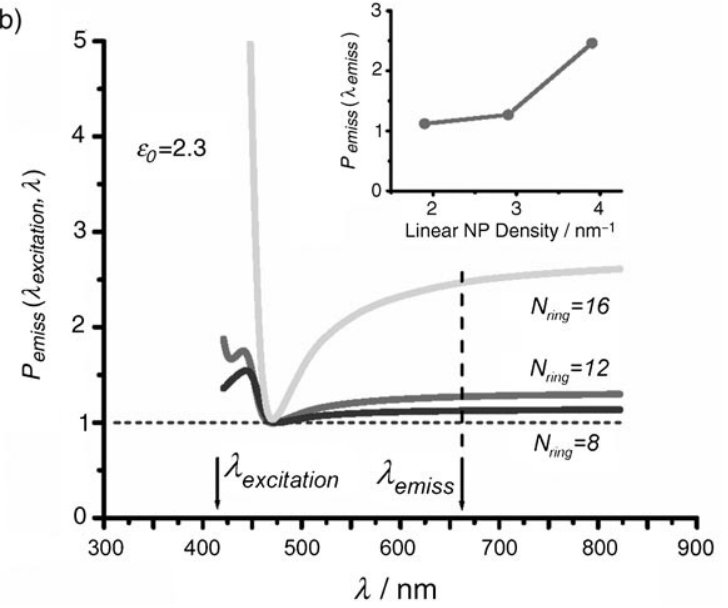

Figure 3. a) Field-enhancement factor for three superstructures with linear NP densities of 1.95, 2.92, and $3.89 \mathrm{~nm}^{-1}$. Inset: Calculated absorption by a single Ag NP. The position of the plasmon resonance corresponds well with the experimental data (Figure 1 b). The two curves for the 12-NP rings correspond to $I_{\max }=1$ and $2(I$ is the spherical harmonic index); the differences are minimal. b) Emissionenhancement factor as a function of the emission wavelength for three superstructures with linear NP densities of $1.95,2.92$, and $3.89 \mathrm{~nm}^{-1}$; the excitation wavelength is taken as $420 \mathrm{~nm}$. Inset: Calculated emission-enhancement factor at an emission wavelength of $660 \mathrm{~nm}$.

5510, and 7391. These numbers are comparable to the NP/NW ratios for solutions $\mathrm{A}$ and $\mathrm{B}$ (Table 1).

The calculations demonstrate (Figure 3a) that the strength of the collective plasmon field increases gradually with increasing density of metal NPs in the shell. The spectral characteristic (energy) of the collective plasmons resonating between many Ag particles in the NP shell of the superstructure becomes wider. The factor $P_{\text {field }}(\lambda)$ is strongly enhanced for $\lambda_{\text {excitation }}$ between 400 and $450 \mathrm{~nm}$ and slightly reduced for $\lambda_{\text {emiss }}$ at around $660 \mathrm{~nm}$; thus the probability of photon emission at $660 \mathrm{~nm}$ in Ag-based structures is not increased but rather is even reduced slightly as a result of dynamic screening inside the shell (Figure 2, inset a). For higher density of NPs, the plasmon peak is located around $425 \mathrm{~nm}$, which is red-shifted relative to the spectrum of individual particles. This shift is illustrated by a comparison of the plasmon resonance in the Ag-NP shell (Figure $3 \mathrm{a}$ ) with the plasmon peak of a single Ag NP (Figure $3 \mathrm{a}$, inset). Again, this change results from the formation of collective plasmon resonance in the Ag-NP shell. This change is in exactly the same spectral region in which the new peak develops in the excitation spectrum (Figure 2). The nice match of the experimental results and calculations demonstrates the validity of the hypothesis of the enhanced absorption.

Some shortening of exciton lifetime of the Ag-based superstructures is observed, as was the case for Au-based superstructures (Figure 1d), although the difference is far less drastic. This effect is attributed mostly to exciton-energy dissipation in the metal. This difference between $\mathrm{Au}-$ and $\mathrm{Ag}$ based structures is the position of the plasmon resonance with respect to the exciton energy in the CdTe NWs. In the AuCdTe complex, the exciton energy is close to the Au plasmon peak. In the Ag-based system, the plasmon resonance is relatively far from the exciton peak. Moreover, the electric field from Ag NPs might activate some other decay mechanisms, such as nonradiative recombination pathways, which are likely to contribute to the reduced lifetime as well.

To evaluate the emission-enhancement effect qualitatively, we calculated the emission-enhancement factor $P_{\text {emiss }}\left(\lambda_{\text {excitation }}, \lambda\right)$ for the emission wavelengths $\lambda>\lambda_{\text {excitation }}$ (Figure $3 \mathrm{~b}$ ). In the experimentally important region around $\lambda_{\text {emiss }}=660 \mathrm{~nm}$, the factor $P_{\text {emiss }}\left(\lambda_{\text {excitation }}, \lambda\right)$ increases rapidly with the number of attached NPs (Figure $3 \mathrm{~b}$, inset). The theoretical factor $P_{\text {emiss }}\left(\lambda_{\text {excitation }}, \lambda\right)$ for a linear NP density of $2.9 \mathrm{~nm}^{-1}$ is more than 2 , which is very similar to actual fluorescence enhancement in Figure 1c. The theoretical estimate from Figure $3 \mathrm{~b}$ (2.5) also compares well with the experimental value of $P_{\text {emiss }}\left(\lambda_{\text {excitation }}, \lambda\right) \approx 3.4$ (derived in the Supporting Information). Thus, the idealized model presented herein gives a very good description of the processes in NP-NW superstructures and metal-semiconductor metamaterials.

In conclusion, we observed a twofold enhancement of luminescence intensity in the nanoscale bioconjugated superstructures made from CdTe NWs and Ag NPs. Theoretical calculations of the electric field in the cylindrically organized NPs and experimental data suggest that the enhancement in emission originates from the increase in absorption of the AgNP shells in the regime of the collective plasmon resonance. This situation is qualitatively different from the PL enhancement in the Au-NP/NW system studied previously. The fundamental importance of these findings is twofold: 1) The results demonstrate metamaterials for which the spatial organization of metal particles has direct consequences on the optical properties as a result of the collective nature of interactions. 2) The described calculation method can be used to predict properties of nanoscale superstructures. From a practical point of view, the combination of Au and Ag NPs may lead to the enhancement of both absorption and emission in semiconductor nanostructures, which could be utilized in a variety of optoelectronic or energy-conversion devices, for example, in solar-energy devices. 


\section{Experimental Section}

CdTe NPs and NWs were prepared as described in detail elsewhere. ${ }^{[27,34]} \mathrm{Ag}$ NPs were synthesized in solution from $\mathrm{AgNO}_{3}$ $\left(\right.$ Aldrich $\left.^{[35]}\right)$, soluble starch $\left(\right.$ Aldrich $\left.^{[35]}\right)$, and D-glucose $\left(\right.$ Aldrich $\left.^{[35]}\right) \cdot{ }^{[36]}$ Ethylene dichloride (EDC) and sulfo- $N$-hydroxysuccinimide (NHS) were used as zero-length cross-links to bind the inorganic and biological materials covalently (Ag NPs with streptavidin (SA), CdTe NWs with D-biotin). ${ }^{[1,37]}$ Ag NPs were bioconjugated with SA as follows: unbound starches in the Ag-NP solution were removed by repeated centrifugation and redispersion until a clear and transparent Ag-NP solution was obtained. An Ag-NP dispersion $(1 \mathrm{~mL})$ was mixed with thioglycolic acid $(8.7 \mu \mathrm{M})$ for $24 \mathrm{~h}$. The EDC and NHS procedures were followed to activate the carboxylic acid groups. Spectra of the bioconjugates were measured with a UV/Vis spectrophotometer (Agilent, Model-8453 ${ }^{[35]}$ ). The luminescence and excitation spectra of the NP-NW dispersions were measured on a Fluoromax-3 spectrofluorometer (Jobin Yvon/SPEX Horiba $^{[35]}$ ) every 1-2 minutes for up to 40 minutes. Atomic force microscopy (AFM) ${ }^{[35]}$ and JEOL 2010F TEM ${ }^{[35]}$ (with an accelerator voltage of $200 \mathrm{kV}$ ) were used to observe the morphology of the bioconjugates of Ag NPs and CdTe NWs. The lifetimes of the respective nanomaterials were measured with a Fluorolog Tau-3 (Jobin Yvon/SPEX Horiba ${ }^{[35]}$ ).

Received: January 26, 2006

Published online: June 27, 2006

Keywords: bioconjugation - luminescence - metamaterials . nanotechnology $\cdot$ silver

[1] J. Lee, A. O. Govorov, J. Dulka, N. A. Kotov, Nano Lett. 2004, 4, $2323-2330$.

[2] E. Prodan, C. Radloff, N. J. Halas, P. Nordlander, Science 2003, 302, 419-422.

[3] X. Michalet, F. Pinaud, T. D. Lacoste, M. Dahan, M. P. Bruchez, A. P. Alivisatos, S. Weiss, Single Mol. 2001, 2, 261-276.

[4] J. Zhang, N. Coombs, E. Kumacheva, Y. Lin, E. H. Sargent, $A d v$. Mater. 2002, 14, $1756-1759$.

[5] Z. Li, R. Jin, C. A. Mirkin, R. L. Letsinger, Nucleic Acids Res. 2002, 30, $1558-1562$.

[6] S. Westenhoff, N. A. Kotov, J. Am. Chem. Soc. 2002, 124, $2448-$ 2449.

[7] G. P. Goodrich, M. R. Helfrich, J. J. Overberg, C. D. Keating, Langmuir 2004, 20, 10246-10251.

[8] J. Jiang, K. Bosnick, M. Maillard, L. Brus, J. Phys. Chem. B 2003, 107, $9964-9972$.

[9] K. Kneipp, Y. Wang, H. Kneipp, L. T. Perelman, I. Itzkan, R. R. Dasari, M. S. Feld, Phys. Rev. Lett. 1997, 78, 67-1670.

[10] M. Maillard, P. Huang, L. Brus, Nano Lett. 2003, 3, 11-1615.

[11] S. Riikonen, I. Romero, F. J. Garcia de Abajo, Phys. Rev. B 2005, $71,5104$.

[12] I. Willner, B. Willner, Pure Appl. Chem. 2002, 74, 73-1783.

[13] A. L. Rogach, Angew. Chem. 2003, 115, 150-151; Angew. Chem. Int. Ed. 2003, 42, 148-149.

[14] Y. Lin, H. Skaff, T. Emrick, A. D. Dinsmore, T. P. Russell, Science 2003, 299, 226-229.

[15] S. Chen, K. Kimura, Chem. Lett. 1999, 233-234.

[16] E. V. Shevchenko, D. V. Talapin, S. O'Brien, C. B. Murray, J. Am. Chem. Soc. 2005, 127, 8741-8747.

[17] S. Zhang, W. Fan, N. C. Panoiu, K. J. Malloy, R. M. Osgood, S. R. J. Brueck, Phys. Rev. Lett. 2005, 95, 137404.

[18] T. Vo-Dinh, Trends Anal. Chem. 1998, 17, 557-582.

[19] K. G. Thomas, P. V. Kamat, Acc. Chem. Res. 2003, 36, 888-898.

[20] Y. C. Cao, R. Jin, C. A. Mirkin, Science 2002, 297, 1536-1540.
[21] S. Link, M. A. El Sayed, Annu. Rev. Phys. Chem. 2003, 54, 331 366.

[22] A. N. Shipway, E. Katz, I. Willner, ChemPhysChem 2000, 1, 18 52.

[23] J. D. Baena, R. Marques, F. Medina, J. Martel, Phys. Rev. B 2004, 69, 014402.

[24] R. Marques, F. Medina, R. Rafii-El-Idrissi, Phys. Rev. B 2002, 65, 144440.

[25] A. O. Govorov, W. Zhang, T. Skeini, H. Richardson, J. Lee, N. A. Kotov, Nanoscale Res. Lett. 2005, 1, 100101.

[26] J. Lee, A. O. Govorov, N. A. Kotov, Angew. Chem. 2005, 117, 7605-7608; Angew. Chem. Int. Ed. 2005, 44, 7439-7442.

[27] Z. Tang, N. A. Kotov, M. Giersig, Science 2002, 297, 237-240.

[28] Z. Liu, H. Li, H. Wang, D. Shen, X. Wang, P. F. A. Alkemade, J. Mater. Res. 2000, 15, $1245-1247$.

[29] L. M. Liz-Marzan, P. Mulvaney, J. Phys. Chem. B 2003, 107, $7312-7326$

[30] H. C. van de Hulst, Light Scattering by Small Particles, John Wiley \& Sons, New York, 1957, p. 470.

[31] R. M. Penner, J. Phys. Chem. B 2002, 106, 3339-3353.

[32] J. Lee, A. O. Govorov, N. A. Kotov, Nano Lett. 2005, 5, $2063-$ 2069.

[33] E. D. Palik, Handbook of Optical Constants of Solids, Academic Press, Orlando, 1985, p. 804.

[34] N. Gaponik, D. V. Talapin, A. L. Rogach, K. Hoppe, E. V. Shevchenko, A. Kornowski, A. Eychmueller, H. Weller, J. Phys. Chem. B 2002, 106, 7177-7185.

[35] Certain commercial equipment, instruments, or materials are identified in this paper to specify the experimental procedure adequately. Such identification is not intended to imply recommendation or endorsement by the National Institute of Standards and Technology, nor is it intended to imply that the materials or equipment identified are necessarily the best available for the purpose.

[36] P. Raveendran, J. Fu, S. L. Wallen, J. Am. Chem. Soc. 2003, 125, $13940-13941$.

[37] N. N. Mamedova, N. A. Kotov, A. L. Rogach, J. Studer, Nano Lett. 2001, 1, 281-286. 